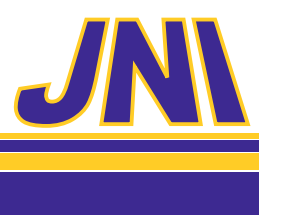

JOURNAL OF

NEUROINFLAMMATION

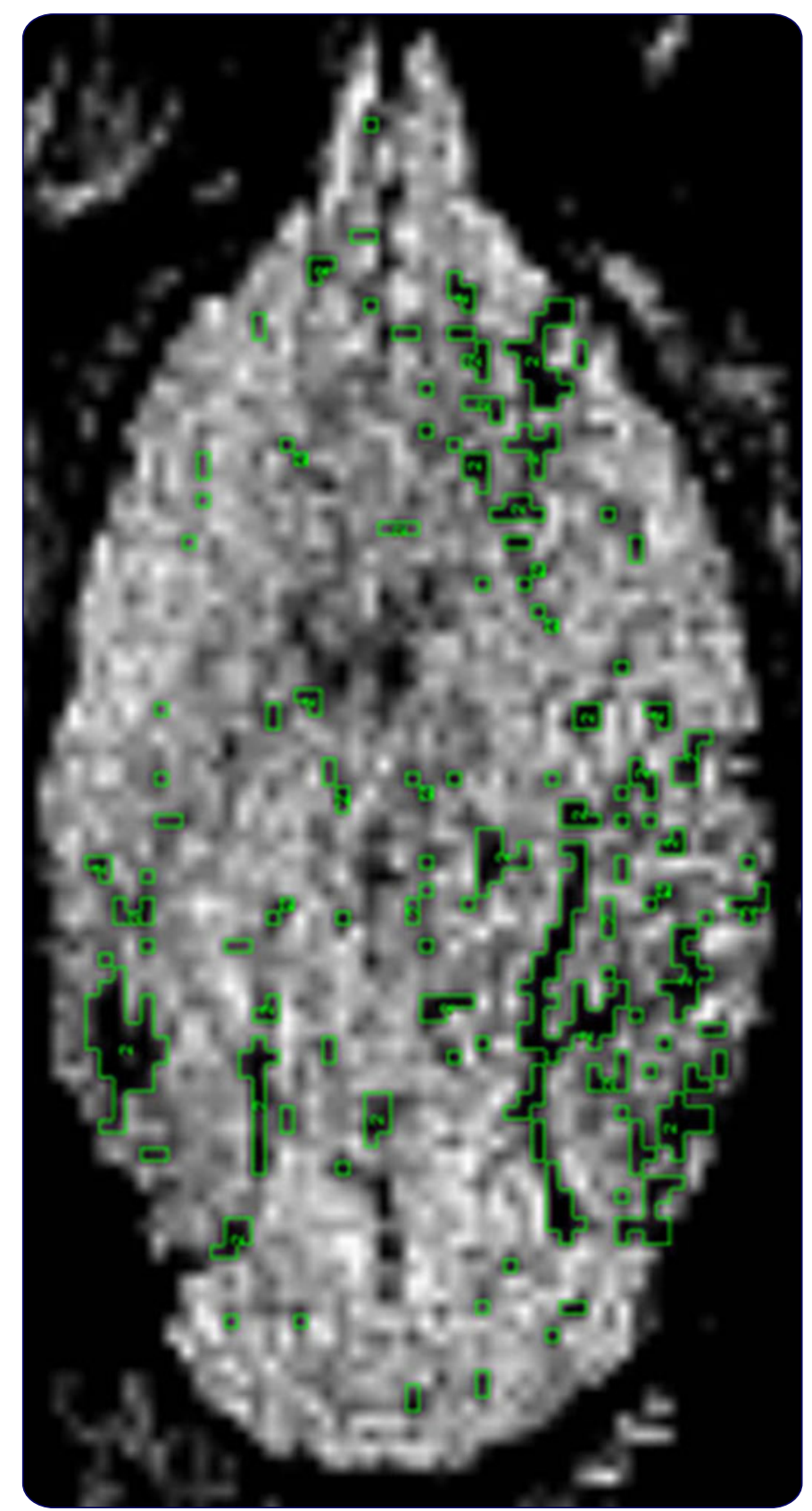

\title{
Preserved vascular integrity and enhanced survival following neuropilin-1 inhibition in a mouse model of CD8 T cell-initiated CNS vascular permeability
}

Suidan et al. 


\title{
Preserved vascular integrity and enhanced survival following neuropilin-1 inhibition in a mouse model of CD8 T cell-initiated CNS vascular permeability
}

Georgette L Suidan ${ }^{1,2+}$, Jonathan W Dickerson ${ }^{1,2+}$, Holly L Johnson ${ }^{4,5}$, Theresa W Chan ${ }^{1}$, Kevin D Pavelko ${ }^{5}$, Istvan Pirko ${ }^{2,3}$, Kim B Seroogy ${ }^{1,2}$ and Aaron J Johnson ${ }^{1,4,5^{*}}$

\begin{abstract}
Background: Altered permeability of the blood-brain barrier (BBB) is a feature of numerous neurological conditions including multiple sclerosis, cerebral malaria, viral hemorrhagic fevers and acute hemorrhagic leukoencephalitis. Our laboratory has developed a murine model of CD8 T cell-initiated central nervous system (CNS) vascular permeability in which vascular endothelial growth factor (VEGF) signaling plays a prominent role in BBB disruption.

Findings: In this study, we addressed the hypothesis that in vivo blockade of VEGF signal transduction through administration of peptide (ATWLPPR) to inhibit neuropilin-1 (NRP-1) would have a therapeutic effect following induction of CD8 T cell-initiated BBB disruption. We report that inhibition of NRP-1, a co-receptor that enhances VEGFR2 (flk-1) receptor activation, decreases vascular permeability, brain hemorrhage, and mortality in this model of CD8 T cell-initiated BBB disruption. We also examine the expression pattern of VEGFR2 (flk-1) and VEGFR1 (flt-1) mRNA expression during a time course of this condition. We find that viral infection of the brain leads to increased expression of flk-1 mRNA. In addition, flk-1 and flt-1 expression levels decrease in the striatum and hippocampus in later time points following induction of CD8 T cell-mediated BBB disruption.
\end{abstract}

Conclusion: This study demonstrates that NRP-1 is a potential therapeutic target in neuro-inflammatory diseases involving BBB disruption and brain hemorrhage. Additionally, the reduction in VEGF receptors subsequent to BBB disruption could be involved in compensatory negative feedback as an attempt to reduce vascular permeability.

Keywords: Vascular endothelial growth factor (VEGF), CD8 T cell, CNS vascular permeability, Blood-brain barrier (BBB), Neuropilin-1 (NRP-1), Fetal liver kinase 1 (flk-1), FMS-related tyrosine kinase-1 (flt-1)

\section{Findings}

Disruption of the blood-brain barrier (BBB) is a hallmark feature of numerous neurological disorders as diverse as multiple sclerosis, stroke, epilepsy, infection, cerebral malaria and acute hemorrhagic leukoencephalitis (AHLE) [1-4]. Immune cells have been linked to central nervous system (CNS) vascular permeability and the ensuing neuropathology in all of the aforementioned conditions. Therefore, defining mechanisms by which

\footnotetext{
* Correspondence: johnson.aaron2@mayo.edu

${ }^{\dagger}$ Equal contributors

${ }^{1}$ Neuroscience Graduate Program, University of Cincinnati, Cincinnati, $\mathrm{OH}$ 45267, USA

${ }^{4}$ Department of Neurology, Mayo Clinic, Rochester, MN 55905, USA Full list of author information is available at the end of the article
}

immune cells promote BBB disruption is of paramount importance for understanding numerous neurologic diseases and developing therapeutic strategies to treat or prevent them. One cytokine, vascular endothelial growth factor (VEGF), has been strongly implicated in vascular permeability. Nevertheless, a complete mechanism by which VEGF contributes to BBB dysregulation under neuro-inflammatory conditions has yet to be elucidated [5]. VEGF has been implicated in the vascular permeability condition associated with Dengue hemorrhagic fever (DHF) as well as cerebral malaria [6,7]. Signaling by VEGF occurs via activation of its high affinity receptors. Among these, VEGF receptor flk-1 is thought to play the most prominent role in angiogenesis and vascular permeability as it is highly expressed on cerebral 
endothelial cells. Upon binding by VEGF, flk-1 undergoes phosphorylation at several tyrosine residues achieving an activated state [8]. Neuropilin-1 (NRP-1), a VEGF co-receptor, is a non-tyrosine kinase transmembranous glycoprotein that enhances the interaction of VEGF with flk-1 and amplifies the angiogenic effects of this signal transduction [9-11].

To study the interaction between antigen-specific CD8 $\mathrm{T}$ cells and the neurovascular unit (NVU) under neuroinflammatory conditions, our laboratory has developed an in vivo model using a variation of the Theiler's murine encephalomyelitis virus (TMEV) infection commonly used to study multiple sclerosis [12-15]. Through the use of this model system, we recently reported that VEGF mRNA is expressed predominantly in neurons, as early as two hours post-induction of CD8 T cell-initiated permeability. Detectable signal transduction was observed with phosphorylation of VEGF receptor flk-1 significantly increasing shortly thereafter. In these studies, we determined that inhibition of neuropilin-1 prevented increased phosphorylation of flk-1, reduced CNS vascular permeability, and preserved microvessel protein levels of the BBB tight junction protein, occludin. These observations supported a hypothesis in which CD8 T cell-initiated BBB disruption was occurring through neuronal expression of VEGF, VEGF signal transduction, and ultimately ablation of $\mathrm{BBB}$ tight junctions in CNS microvessels [16]. In the current study, we assessed flk-1 and flt-1 mRNA expression in the brain during the course of CD8 T cell-initiated CNS vascular permeability. We also determined the extent by which neuropilin1 receptor inhibition reduces vascular permeability and hemorrhage formation as measured by gadoliniumenhanced T1-weighted and T2\%-weighted magnetic resonance imaging (MRI), respectively.

CNS vascular permeability was induced as described previously [12]. Briefly, C57BL/6 mice were infected intracranially with $2 \times 10^{6}$ PFU Daniel's strain of TMEV. Seven days post-TMEV infection, mice were injected intravenously with $0.1 \mathrm{mg} \mathrm{VP2} 2_{121-130}$ (FHAGSLLVFM) peptide (GenScript Corp. Piscataway, NJ, USA) to initiate CD8 T cell-initiated BBB disruption. We have previously published that virus infection alone is not sufficient to induce overt BBB disruption. Seven-day TMEV-infected mice have minimal CNS vascular permeability, normal BBB tight junctions, and lack microhemorrhages [14-16]. Mice were euthanized at various time points following this induction to analyze gene expression events, vascular permeability, hemorrhage and overall survival. All experiments were approved by the Institutional Animal Care and Use Committee of the University of Cincinnati.

To determine the contribution of neuropilin-1 inhibition in reducing CNS vascular permeability, microhemorrhage, and overall survival, $3 \mathrm{mg}$ of ATWLPPR peptide or PBS (sham treatment) was intravenously injected at $-30 \mathrm{~min}$ utes, 3 hours, 6 hours and 9 hours post-administration of VP2 $121-130$ to initiate CD8 T cell-initiated BBB disruption. Twenty-four hours post-VP2 $2_{121-130}$ peptide administration, mice were scanned using gadolinium-enhanced T1weighted MRI to assess CNS vascular permeability and T2/T2*-weighted MRI to assess hemorrhage formation according to our previously published methods [14]. Analyze 10 software developed by the Mayo Clinic was used to quantify the three-dimensional volume of gadolinium leakage from vasculature as well as the volume of microhemorrhage. Treatment with NRP-1 inhibitor $(\mathrm{n}=4)$ markedly reduced three-dimensional gadolinium enhancement leakage when compared to PBS-treated controls $(n=2)(P<0.001$, Student's $t$-test) (Figure 1A-C). Treatment with NRP-1 inhibitor $(n=5)$ also significantly reduced microhemorrhage formation when compared to treatment with $\operatorname{PBS}(\mathrm{n}=4) \quad(P=0.023$, Student's $t$-test $)$ (Figure 1D-H). In addition, inhibiting NRP-1 significantly increased survival of mice administered VP2 $121-130$ peptide undergoing CD8 $\mathrm{T}$ cell-initiated $\mathrm{BBB}$ disruption $(P=0.014$, Kaplan-Meier survival curve analysis). Mice were monitored for 72 hours post-administration of VP2 ${ }_{121-130}$ peptide and did not receive additional NRP-1 inhibitor treatment past the 9-hour time point. We also determined that treatment with NRP-1 inhibitor did not alter viral loads when compared to treatment with PBS using quantitative real time (RT)-PCR to detect viral RNA as a ratio to actin mRNA (PBS group mean = $57027 \pm$ SD 12287, $3 \mathrm{mg}$ NRP-1 inhibitor mean $=43687$ $\pm 15474, P=0.519$, Student's $t$-test). Additionally, vascular permeability was analyzed by quantifying fluorescein isothiocyanate (FITC)-albumin leakage into the brain in mice administered normal goat serum $(\mathrm{n}=2 ; 0.750 \mathrm{mg})$, DC101 antibody to flk-1 $(\mathrm{n}=4 ; 0.500 \mathrm{mg})$, or DC6.12 antibody to flt- $1(\mathrm{n}=4 ; 0.750 \mathrm{mg}) 2$ days prior, to determine the contribution of VEGF receptors to CNS vascular permeability. We determined that pre-treatment with DC101, but not DC6.12, significantly reduced CNS vascular permeability when compared to treatment with normal goat serum $(P=0.003)$ (Figure 1I). This further supports our hypothesis that VEGF plays a role in BBB disruption through binding receptor flk-1.

To further extend our previous study [16], we performed an analysis of VEGF receptor gene expression following induction of CD8 T cell-initiated BBB disruption. In situ hybridization was carried out on fresh-frozen, cryostat-cut (at 10- $\mu \mathrm{m}$ thickness), slide-mounted sections throughout the brain. Semi-adjacent sections were hybridized with ${ }^{35} \mathrm{~S}$-labeled cRNA sense (control) and anti-sense probes for detection and localization of VEGF receptors flk-1 and flt- 1 mRNAs according to our previously published protocol $[16,17]$. The flk-1 and flt-1 

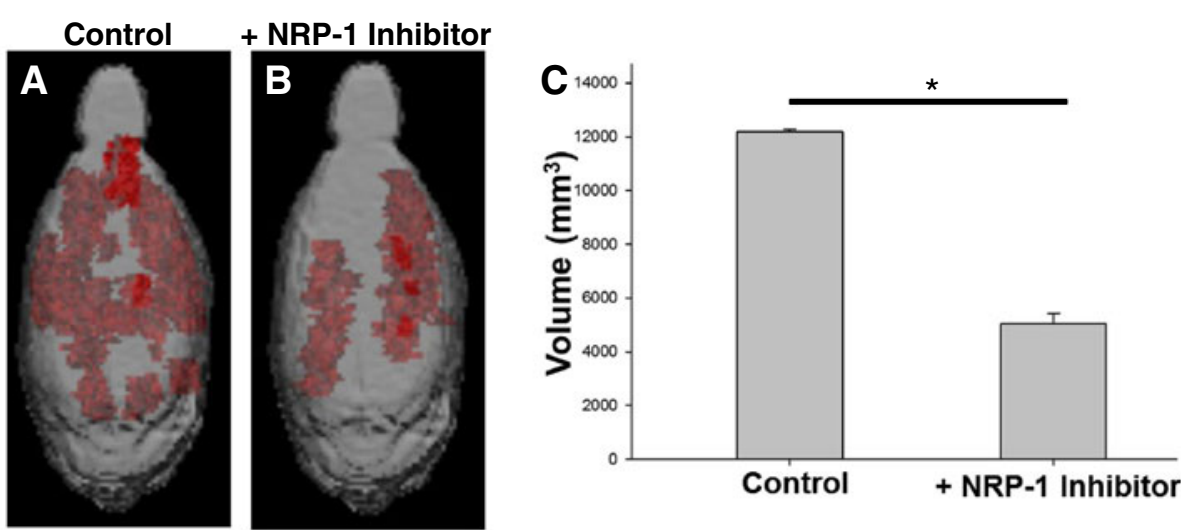

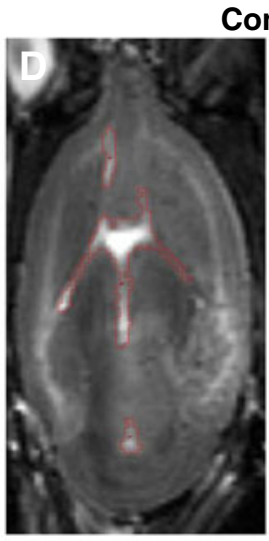

Control

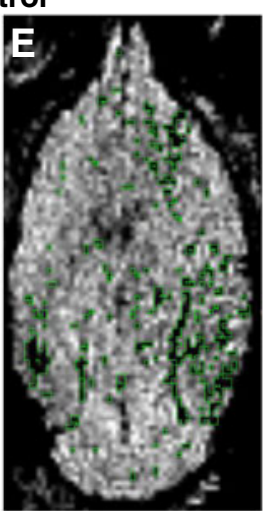

H

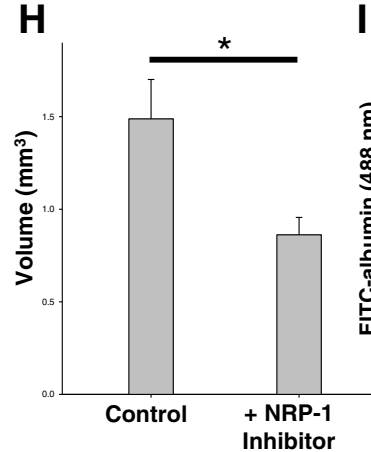

I

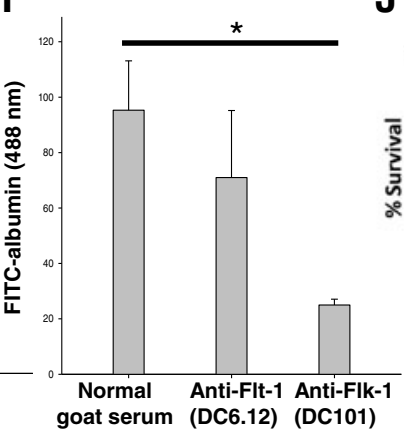

J
+ NRP-1 Inhibitor
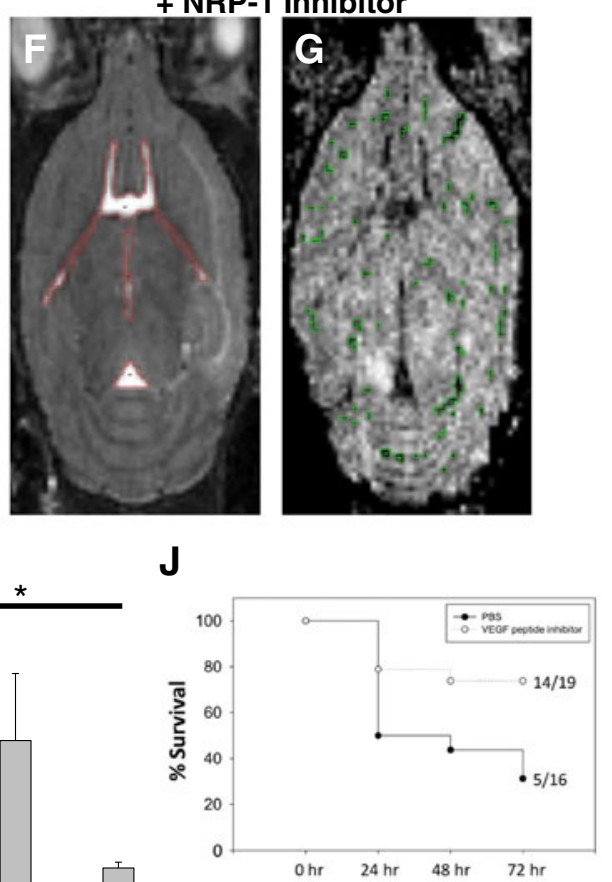

Hours post administration of VP2 peptide

Figure 1 NRP-1 inhibition reduces central nervous system (CNS) vascular permeability, microhemorrhage formation, and morbidity following induction of CD8 T cell-initiated blood-brain barrier (BBB) disruption. We present the three-dimensional volume of gadolinium leakage as measured using T1-weighted magnetic resonance imaging (MRI) in a representative animal treated with (A) phosphate-buffered saline (PBS) or (B) NRP-1 inhibitor, 24 hours post-induction of vascular permeability with intravenous injection of VP2 $121-130$ peptide. In (C), we demonstrate reduced three-dimensional volumes of gadolinium leakage in animals receiving NRP-1 inhibitor as compared to sham PBS-treated controls $(P<0.001)$. T2 MRI was performed on animals receiving $(\mathbf{D})$ sham PBS treatments or $(\mathbf{F})$ NRP-1 inhibitor. Following these scans, the ventricle size was determined (red outline) and subsequently not included in the analysis of microhemorrhage area (green outlines) determined in (E) PBS-treated and (G) NRP-1 inhibitor-treated groups analyzed by T2*-weighted MRI. Using this method of analysis, we observed reduced microhemorrhage in NRP-1 inhibitor-treated animals as compared to PBS-treated controls $(P=0.023)$. All MRI scans were analyzed blind before breaking the code of each treatment group. (I) Quantification of FITC-albumin leakage into the brain reveals that pretreatment with DC101 antibody to flk-1, but not DC6.12 antibody to flt-1, is effective in reducing CNS vascular permeability $(P=0.003)$. In (J), administration of NRP-1 inhibitor enhances survival of mice undergoing CD8 T cell-initiated CNS vascular permeability $(P=0.014)$.

cDNA plasmids were contained in a pGEM3 vector and consisted of $390 \mathrm{bp}$ and $660 \mathrm{bp}$, respectively (kindly provided by LF Brown, Harvard University [18]). Labeled probes were prepared by in vitro transcription from linearized cDNA plasmids using the proper RNA polymerase in the presence of excess ${ }^{35} \mathrm{~S}$-UTP (PerkinElmer, Waltham MA, USA) and were generated as previously described [19]. The pretreated sections were 
incubated overnight at $60{ }^{\circ} \mathrm{C}$ in hybridization solution consisting of $50 \%$ de-ionized formamide, $10 \%$ dextran sulfate, $20 \mathrm{mM}$ Tris- $\mathrm{HCl}, 1 \mathrm{mM}$ EDTA, $1 \mathrm{X}$ Denhardt's solution, $0.33 \mathrm{mg} / \mathrm{ml}$ denatured salmon sperm DNA, $0.15 \mathrm{mg} / \mathrm{ml}$ tRNA, $40 \mathrm{mM}$ dithiothreitol, DEPC $\mathrm{H}_{2} \mathrm{O}$ and the ${ }^{35} \mathrm{~S}$-labeled probe at a concentration of $1 \times 10^{6} \mathrm{cpm} / 50 \mu \mathrm{l}$. After hybridization, sections were washed in a series of standard saline citrate washes including a ribonuclease A treatment. Slides were then exposed to BioMax MR film (Kodak, Rochester NY, USA) for 8 days for generation of film autoradiographs. The films were developed with Kodak GPX developer and fixer. Semi-quantitative analysis of the dorsal hippocampus (including the dentate gyrus granule cell layer, hippocampal principal cell layers and molecular layers) and striatum ipsilateral to the hemisphere of TMEV

\section{A}

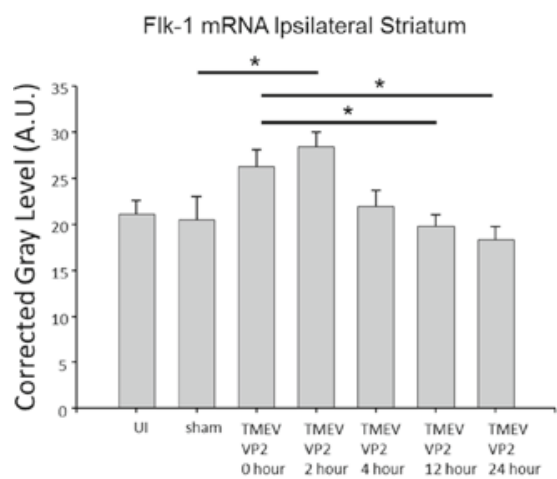

C

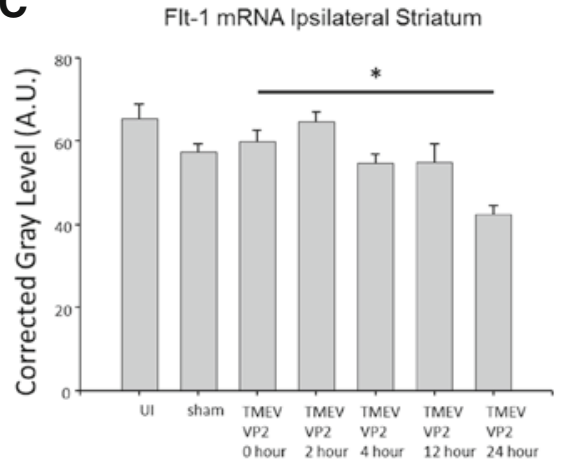

$\mathbf{E}$

을
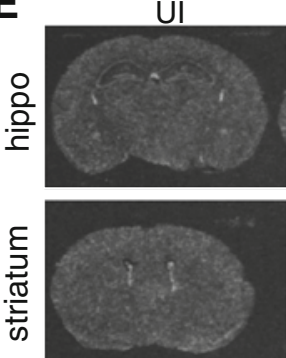

B

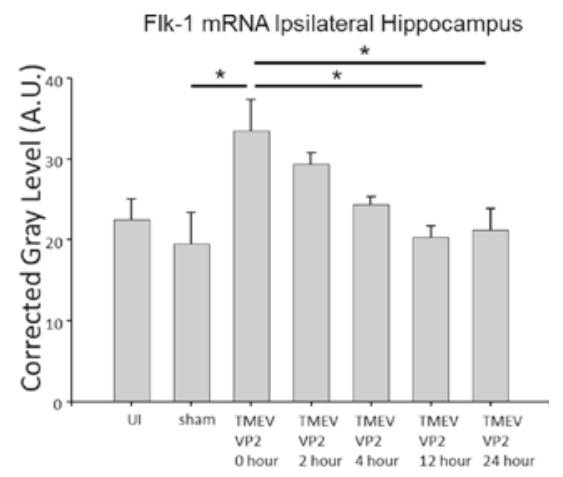

D
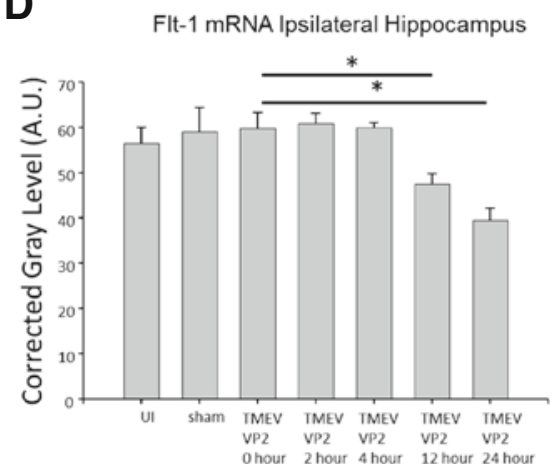

$4 \mathrm{~h}$

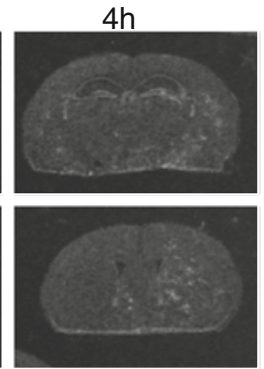

$24 \mathrm{~h}$

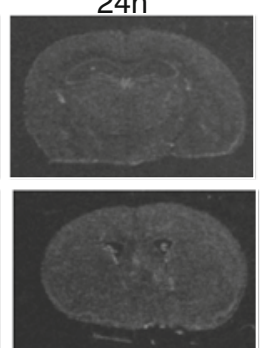

Figure 2 Analysis of vascular endothelial growth factor (VEGF) receptors flk-1 and flt-1 mRNA expression in brain using in situ hybridization. Flk-1 and flt-1 mRNA hybridization was determined in uninfected, sham-infected, Theiler's murine encephalomyelitis virus (TMEV)infected animals, and in mice at 2, 4, 12 and 24 hours post-induction of CD8 T cell-initiated BBB disruption through administration of VP2 121-130 peptide. We present semiquantitative analysis of flk-1 expression in (A) the striatum and (B) the hippocampus of uninfected, saline-treated (sham), TMEV-infected and VP2 $2_{121-130}$ peptide-administered animals. Also shown is semiquantitative analysis of flt-1 expression in (C) the striatum and (D) the hippocampus of uninfected, phosphate-buffered saline (PBS)-treated (sham), TMEV-infected and VP2 $2_{121-130}$ peptide-administered animals undergoing CD8 T cell-initiated BBB disruption $(P<0.05)$. (E) Representative film autoradiographs depicting flk-1 mRNA expression in the hippocampus (top row) and striatum (bottom row) of TMEV-infected mice. The expression of flk-1 mRNA appears to be altered in TMEV-injected animals prior to induction of vascular permeability ( 0 hours). Flk-1 mRNA levels then decline by 4 hours after induction of vascular permeability, and returned to normal levels by 24 hours (compare $24 \mathrm{~h}$ with $\mathrm{UI}$ ). Arrows indicate the hippocampus (top panel) or the striatum (bottom panel). h, hours; Ul, uninfected. 
infection was performed using optical density measurements (Scion Image software, National Institutes of Health). The corrected grey levels were generated by subtracting a background measurement (taken from a non-tissue-containing area on the same slide) from the optical density measurements in the hippocampus or striatum for each section. No specific labeling was obtained with the control sense riboprobes. Mean and standard error values for in situ hybridization measurements were calculated using software program SigmaStat (SYSTAT Software Inc, San Jose CA, USA). Bar graphs with standard error values were plotted on software program SigmaPlot (SYSTAT Software Inc).

In Figure $2 \mathrm{~A}$ and $\mathrm{B}$, we demonstrate that at seven days post-TMEV infection, there is significantly increased expression of flk-1 mRNA in the hippocampus ( $\mathrm{n}=4$ for both sham and TMEV infected animals, $P=0.016$ ) and striatum $(\mathrm{n}=4$ for both sham and TMEV infected animals; $P=0.025)$ when compared to sham controls, in which sterile PBS is intracranially administered to the brain. These data also demonstrate that flk-1 mRNA expression is decreased in the hippocampus $(\mathrm{n}=4$; $P=0.005)$ and striatum $(\mathrm{n}=4 ; P=0.009)$ by 12 hours post-administration of $\mathrm{VP} 2121-130$ peptide, returning to similar expression levels as sham-treated animals. Unlike flk-1 mRNA expression, flt-1 mRNA expression in the hippocampus and striatum remains unaltered 7 days post-TMEV infection (Figure $2 \mathrm{C}$ and $\mathrm{D}$ ). However, flt-1 mRNA expression is significantly decreased in the hippocampus by 12 hours post-administration of VP2 121-130 $(\mathrm{n}=4 ; P=0.029$; Figure $2 \mathrm{D})$ and remained significantly decreased at 24 hours $(\mathrm{n}=4 ; P=0.002)$. By 24 hours, flt- 1 expression is significantly decreased in the striatum (sham group $n=4,24$ hour group $n=4 ; P<0.001$; Figure 2C). We have previously published that vascular permeability occurs as early as 4 hours post administration of VP2 peptide proportionally with the increase in VEGF cytokine expression [16]. Also in this previous study, flk-1 receptor becomes phosphorylated at 4 hours post VP2 peptide administration [16]. The reduction of flk-1 and flt-1 mRNA expression observed in this study are therefore indicative of negative regulation of VEGF receptor gene expression following increased levels of VEGF cytokine and ensuing BBB disruption in these animals.

Using this model system of immune-mediated BBB disruption, we have demonstrated that intracranial TMEV infection alters expression of flk-1, but not flt-1, mRNA in the CNS. We also show that inhibition of the VEGF co-receptor, neuropilin-1, improves survival outcome in this model. The observation that VEGF receptors contribute to vascular permeability is prominent in the literature. Studies in DHF patients have shown that vascular permeability is inversely correlated to the amount of soluble VEGFR2, the human homolog of flk-1. Levels of plasma-soluble VEGFR1, the human homolog of flt-1, were stable indicating that VEGFR2 is the key receptor involved in DHF [7]. Based on the results obtained in our model system, we hypothesize that flk-1 mRNA expression is upregulated during viral infection to promote angiogenesis and vascular permeability to enable effective inflammation necessary to clear pathogens. Furthermore, this study provides in vivo evidence that NRP-1, a VEGF co-receptor that has been shown to enhance flk-1 activity, is a potential target when designing therapies for neuroinflammatory CNS conditions in which BBB integrity is compromised via VEGF-mediated mechanisms.

\section{Abbreviations}

BBB: blood-brain barrier; AHLE: acute hemorrhagic leukoencephalomyelitis; VEGF: vascular endothelial growth factor; NRP-1: neuropilin-1; flk-1: fetal liver kinase 1; flt-1: FMS-related tyrosine kinase-1; TMEV: Theiler's murine encephalomyelitis virus.

\section{Competing interests}

The authors declare that they have no competing interests.

\section{Authors' contributions}

GLS conceived of the study, participated in design, coordination and conception of the study, drafted the manuscript, collected samples for in situ hybridization and optimized NRP-1 inhibitor dosing for survival, hemorrhage and permeability studies. JWD participated in design and coordination of the study and performed and analyzed the in situ hybridization experiments. HLJ performed volumetric image analysis of microhemorrhage and permeability. TWC executed experiments pertaining to NRP-1 inhibition and small animal MRI to detect CNS vascular permeability and microhemorrhage formation. IP participated in design and conception of the study, participated in experimental design of MRI studies. KBS participated in design and conception of the study, helped revise the manuscript for important intellectual content, and participated in the methodology of in situ hybridization experimentation. KDP evaluated viral loads in CNS tissue. AJJ oversaw all aspects of experimental design, execution of experiments, data analysis, and manuscript preparation. This included participating in design, coordination and conception of the study, preparation and revision of the manuscript for important intellectual content, participation in sample collection for in situ hybridization, and assistance with survival studies and MRI for detection of hemorrhage and permeability.

\section{Acknowledgements}

We would like to thank Dr. Lawrence Brown of Harvard for providing the flk1 and flt-1 plasmids required to perform the in situ hybridization analysis. We would also like to thank Mike Bell of Mayo Clinic Immunology for technical assistance with quantitative RT-PCR for detection of virus loads. The authors have no conflicting financial interests. This work is supported by the National Institutes of Health grant NS060881 (AJJ), the Selma Schottenstein Harris

Laboratory for Research in Parkinson's (KBS), and a Scottish Rite

Schizophrenia Foundation Fellowship (JWD).

\section{Author details}

${ }^{1}$ Neuroscience Graduate Program, University of Cincinnati, Cincinnati, $\mathrm{OH}$ 45267, USA. ${ }^{2}$ Department of Neurology, University of Cincinnati College of Medicine, Cincinnati, OH 45267, USA. ${ }^{3}$ Department of Neuroscience, Mayo Clinic, Rochester, MN 55905, USA. ${ }^{4}$ Department of Neurology, Mayo Clinic, Rochester, MN 55905, USA. ${ }^{5}$ Department of Immunology, Mayo Clinic, Gugenheim Building 4-11C, 200 First St. SW, Rochester, MN 55905, USA.

Received: 21 May 2012 Accepted: 18 August 2012

Published: 18 September 2012

\section{References}

1. Weiss N, Miller F, Cazaubon S, Couraud PO: The blood-brain barrier in brain homeostasis and neurological diseases. Biochim Biophys Acta 2009, 1788:842-857. 
2. Kabakus N, Gurgoze MK, Yildirim H, Godekmerdan A, Aydin M: Acute hemorrhagic leukoencephalitis manifesting as intracerebral hemorrhage associated with herpes simplex virus type I. J Trop Pediatr 2005, 51:245-249.

3. Brown H, Hien TT, Day N, Mai NT, Chuong LV, Chau TT, Loc PP, Phu NH, Bethell D, Farrar J, Gatter K, White N, Turner G: Evidence of blood-brain barrier dysfunction in human cerebral malaria. Neuropathol Appl Neurobiol 1999, 25:331-340.

4. Suidan GL, Pirko I, Johnson AJ: A potential role for CD8+ T-cells as regulators of CNS vascular permeability. Neurol Res 2006, 28:250-255.

5. Antonetti DA, Barber AJ, Hollinger LA, Wolpert EB, Gardner TW: Vascular endothelial growth factor induces rapid phosphorylation of tight junction proteins occludin and zonula occluden 1. A potential mechanism for vascular permeability in diabetic retinopathy and tumors. J Biol Chem 1999, 274:23463-23467.

6. Deininger MH, Winkler S, Kremsner PG, Meyermann R, Schluesener HJ: Angiogenic proteins in brains of patients who died with cerebral malaria. J Neuroimmunol 2003, 142:101-111.

7. Srikiatkhachorn A, Ajariyakhajorn C, Endy TP, Kalayanarooj S, Libraty DH, Green S, Ennis FA, Rothman AL: Virus-induced decline in soluble vascular endothelial growth receptor 2 is associated with plasma leakage in dengue hemorrhagic Fever. J Virol 2007, 81:1592-1600.

8. Olsson AK, Dimberg A, Kreuger J, Claesson-Welsh L: VEGF receptor signalling - in control of vascular function. Nat Rev Mol Cell Biol 2006, 7:359-371.

9. Soker S, Miao HQ, Nomi M, Takashima S, Klagsbrun M: VEGF165 mediates formation of complexes containing VEGFR-2 and neuropilin-1 that enhance VEGF165-receptor binding. J Cell Biochem 2002, 85:357-368.

10. Soker S, Takashima S, Miao HQ, Neufeld G, Klagsbrun M: Neuropilin-1 is expressed by endothelial and tumor cells as an isoform-specific receptor for vascular endothelial growth factor. Cell 1998, 92:735-745.

11. Starzec A, Vassy R, Martin A, Lecouvey M, Di Benedetto M, Crepin M, Perret GY: Antiangiogenic and antitumor activities of peptide inhibiting the vascular endothelial growth factor binding to neuropilin-1. Life Sci 2006, 79:2370-2381.

12. Johnson AJ, Mendez-Fernandez Y, Moyer AM, Sloma CR, Pirko I, Block MS, Rodriguez M, Pease LR: Antigen-specific CD8+ T cells mediate a peptide-induced fatal syndrome. J Immunol 2005, 174:6854-6862.

13. Johnson HL, Chen Y, Suidan GL, McDole JR, Lohrey AK, Hanson LM, Jin F, Pirko I, Johnson AJ: A hematopoietic contribution to microhemorrhage formation during antiviral CD8 T cell-initiated blood-brain barrier disruption. Journal of neuroinflammation 2012, 9:60.

14. Pirko I, Suidan GL, Rodriguez M, Johnson AJ: Acute hemorrhagic demyelination in a murine model of multiple sclerosis. Journal of neuroinflammation 2008, 5:31.

15. Suidan GL, McDole JR, Chen Y, Pirko I, Johnson AJ: Induction of blood brain barrier tight junction protein alterations by CD8 T cells. PLoS One 2008, 3:e3037.

16. Suidan GL, Dickerson JW, Chen Y, McDole JR, Tripathi P, Pirko I, Seroogy KB, Johnson AJ: CD8 T cell-initiated vascular endothelial growth factor expression promotes central nervous system vascular permeability under neuroinflammatory conditions. J Immunol 2010, 184:1031-1040.

17. Hemmerle AM, Dickerson JW, Herring NR, Schaefer TL, Vorhees CV, Williams MT, Seroogy KB: (+/-)3,4-methylenedioxymethamphetamine ("Ecstasy") treatment modulates expression of neurotrophins and their receptors in multiple regions of adult rat brain. J Comp Neurol 2012, 520:2459-2474.

18. Detmar M, Velasco P, Richard L, Claffey KP, Streit M, Riccardi L, Skobe M, Brown LF: Expression of vascular endothelial growth factor induces an invasive phenotype in human squamous cell carcinomas. Am J Pathol 2000, 156:159-167.

19. Seroogy KB, Herman JP: In situ hybridization approaches to the study of the nervous system. In Neurochemistry: A Practical Approach. 2nd edition. Edited by Turner AJ, Bachelard HS. Oxford: Oxford University Press; 1997:121-150

\section{doi:10.1186/1742-2094-9-218}

Cite this article as: Suidan et al:: Preserved vascular integrity and enhanced survival following neuropilin-1 inhibition in a mouse model of CD8 T cell-initiated CNS vascular permeability. Journal of Neuroinflammation 2012 9:218.

\section{Submit your next manuscript to BioMed Central and take full advantage of:}

- Convenient online submission

- Thorough peer review

- No space constraints or color figure charges

- Immediate publication on acceptance

- Inclusion in PubMed, CAS, Scopus and Google Scholar

- Research which is freely available for redistribution 